\title{
OWREN'S METHOD FOR THE CONTROL OF ANTICOAGULANT THERAPY
}

\author{
BY \\ M. J. ALLINGTON \\ From the Department of Haematology, Radcliffe Infirmary, Oxford
}

(RECEIVED FOR PUBLICATION OCTOBER 31, 1957)

Anticoagulant therapy is a well-established treatment for coronary thrombosis, pulmonary embolus, and other thrombotic or embolic episodes. The anticoagulant drugs used are usually those of the dicoumarol group. It is known that this group of drugs, "dindevan," "tromexan," " marcoumar," etc., depress the production of prothrombin and possibly to a greater extent that of factor VII (proconvertin). There is a variable time lag between dosage with the particular drug and its effect on the prothrombin and factor VII levels. It is this time lag and the method of estimating the level of the appropriate coagulation factors that have led to some difficulty in controlling the dosage of anticoagulant to be used in individual patients. The Quick one-stage method or some modification of this test has usually been the method of choice in this ccuntry. The two-stage method has also been used. The one-stage methods are sensitive to changes in factors V and VII levels and to a lesser extent of prothrombin; in the two-stage methods factor VII levels are ignored, and this latter fact is of importance, as it is known that factor VII is more markedly reduced than prothrombin. In 1951 Owren and Aas introduced the so-called $P$ and $P$ method (prothrombin and proconvertin) for the control of anticoagulant therapy. The method is sensitive to changes in prothrombin and factor VII levels, but insensitive to changes in factor $\mathrm{V}$ and fibrinogen. The method is basically a onestage one with the following modifications.

(1) The plasma is diluted to increase sensitivity, and this dilution, of course, also minimizes the effect of any heparin in the patient's plasma. (2) Bovine plasma from which the prothrombin and factor VII have been adsorbed is added as a source of fibrinogen and factor V. (3) A standardized saline extract of human brain is used as the thromboplastin. (4) Ionic strength is kept constant by the use of two diluting fluids.

The advantages of this method have been stressed by Owren and Aas (1951), by Astrup,
Müllertz, and Hansen (1951), by Müllertz and $\overrightarrow{-}$ Storm (1954) (who maintained anticoagulant is therapy during major surgery), by Astrup and 을 Müllertz (1954), and by Owren (1954). The advantages claimed by these authors have been: (i) Greatly increased sensitivity over Quick's method, especially in the near normal range, making it very much easier to judge the initial hypocoagulability and subsequent dosage. (ii) There is less random $\vec{\oplus}$ variability than in the unmodified test because 9 irrelevant factors are supplied in standard form. This is very important for the accurate stabilization of patients who will be receiving long-term anticoagulant therapy as out-patients. (iii) The method measures the combined effect of pro- $\stackrel{\mathbb{Q}}{\unrhd}$ thrombin and factor VII, both of which are impor- $\vec{\nexists}$ tant and which may be reduced to a different $\frac{0}{3}$ extent at different stages of treatment. (iv) In Quick's method the optimum calcium concentration may not be used, owing to variations in haematocrit values. This effect is minimized by the dilution. (v) The method is unaffected by small $\frac{5}{3}$ amounts of heparin in the patient's plasma. (vi) The use of a small amount of heparin in the anticoagulant used for the collection of the patient's blood sample enables accurate estima- 윽 tions to be made on blood samples which have $\frac{D}{O}$ been sent through the post. This is also advantageous in the long-term treatment of out-patients. $N$

On the evidence of these observations it was decided that the method should be investigated in this department.

\section{Reagents}

The method given here is the one used routinely in this department and differs slightly from Owren's original method.

A. Stock Solutions.-The following are required :

Owren's Buffer.-Take 5.878 g. sodium diethylbarbiturate, $7.335 \mathrm{~g}$. sodium chloride, and $215 \mathrm{ml}$. $0.1 \mathrm{~N}$ hydrochloric acid, and make up to 1 litre with distilled water. The resultant solution should be of $\bar{\gamma}$ pH 7.33 . 
Citrate Solution.-This is $31.3 \mathrm{~g}$. trisodium citrate (dihydrate) made up to 1 litre with distilled water.

25.6 Molar Trisodium Citrate.-For this $240 \mathrm{ml}$. $3.13 \%$ trisodium citrate solution is made up to 1 litre with distilled water.

One Tenth Molar Calcium Chloride.-See Biggs and Macfarlane (1957).

B. Diluting Solutions.-The first (sol. I) consists of $100 \mathrm{ml} .3 .13 \%$ trisodium citrate solution made up to $700 \mathrm{ml}$. with $0.9 \%$ sodium chloride solution. (This diluting fluid is used only for serial dilutions of pooled normal plasma, before diluting 1 in 10 with diluting fluid B.)

The second (sol. II) contains $200 \mathrm{ml}$. Owren's buffer and $200 \mathrm{ml} .25 .6 \mathrm{M}$. molar trisodium citrate solution made up to 1 litre with $0.9 \%$ sodium chloride solution. (This diluting fluid is used for the 1 in 10 dilution of patient's and normal plasma.)

C. Anticoagulant.-This is made up of $250 \mathrm{ml}$. $3.13 \%$ trisodium citrate solution, $25 \mathrm{mg}$. heparin, and $25 \mathrm{mg}$. merthiolate. All the above solutions should be stored at $4^{\circ} \mathrm{C}$. when not in use.

D. Brain Extract.-A whole fresh human brain is freed of all blood vessels and membranes. The brain matter is emulsified in $1,500 \mathrm{ml}$. of $0.9 \%$ sodium chloride solution which has previously been warmed to a temperature of $37^{\circ} \mathrm{C}$. The emulsification of the brain is best carried out with a "blending machine." We have found the M.S.E. "Ato-mix" very satisfactory for this purpose. The resultant emulsion is centrifuged at 1,800-2,000 r.p.m. for 30 minutes, the supernatant fluid is decanted, the deposit being discarded. Dilutions of a small sample of the supernatant fluid are made in $0.9 \%$ sodium chloride solution. The following dilutions are usually sufficient: $1 / 1,1 / 2$, $1 / 4,1 / 8$. The dilutions are used as thromboplastin in an ordinary one-stage test with normal plasma and M/40 calcium chloride. The bulk of the supernatant fluid is then diluted to correspond with the trial dilution giving the shortest one-stage time. A satisfactory preparation gives one-stage clotting times of 13 to 16 seconds. Finally $10 \%$ of Owren's buffer is added to the brain extract. The extract is then distributed into suitable containers and stored at $-20^{\circ} \mathrm{C}$. The most suitable container for this purpose (and for the bovine plasma, see below) will obviously depend on the requirements of the individual department. We have found that a $5 \mathrm{ml}$. screw-capped Bijou bottle contains sufficient thromboplastin for the number of tests we have to do on a normal day. It is imperative that a fresh bottle of thromboplastin should be used each day, and that once a bottle has been thawed it should not be refrozen for use at a later date.

E. Bovine Plasma.-Bovine blood is collected at the slaughterhouse into $3.5 \%$ aqueous potassium oxalate in the proportion of 1 part of oxalate solution to 9 parts of blood. After thoroughly mixing, the blood is centrifuged to remove the cells. To each 100 mil. of plasma is added $2 \mathrm{~g}$. of citrate-washed barium sulphate (Biggs and Macfarlane, 1957). The barium sulphate plasma suspension is gently agitated for 15 to 20 minutes. At the end of this time the barium sulphate is removed by centrifuging. A second adsorption and very occasionally a third adsorption with similar amounts of barium sulphate is necessary. The plasma should be tested after the removal of the second addition of barium sulphate. A mixture in equal parts of absorbed plasma, brain extract, and calcium chloride should take more than 30 minutes to clot at $37^{\circ} \mathrm{C}$. The clotting time of a similar mixture to which serum diluted 1 in 10 has been added should exceed 90 seconds. Citrated plasma diluted 1 in 10 and added to the mixture should shorten the coagulation time to 40 seconds or less. A coagulation time much greater than 40 seconds probably indicates over-adsorption and will lead to unnecessarily long coagulation times, making the method laborious and time-consuming. The adsorbed plasma is finally stored in a similar manner to the brain extract at $-20^{\circ} \mathrm{C}$. In this department $5 \mathrm{ml}$. amounts are put up in Bijou bottles. It is essential that the plasma is stored in approximately equal amounts.

\section{Technique}

A. Details of the Method.-Blood is collected into the heparin-citrate anticoagulant in the proportion of 1 part of anticoagulant to 9 parts of blood. The plasma is obtained by centrifuging and subsequently diluted 1 in 10 in diluting fluid II. Amounts of $0.2 \mathrm{ml}$. of plasma and $1.8 \mathrm{ml}$. of diluting fluid are suitable for routine use. Two portions, each of $0.1 \mathrm{ml}$. of diluted plasma, are placed in each of two $2 \frac{1}{2}$ in. $\times \frac{3}{8}$ in. test tubes, and $0.1 \mathrm{ml}$. of thromboplastin and $0.1 \mathrm{ml}$. of bovine plasma are added to each tube. The tubes are then placed in a water-bath at $37^{\circ} \mathrm{C}$. for a minimum of three minutes up to a maximum of six minutes. To each tube is added $0.1 \mathrm{ml}$. of previously warmed $\mathrm{CaCl}_{2}$ solution of appropriate concentration. The time taken for the mixture in the tubes to coagulate is recorded. The coagulation time of a normal sample by this method is usually between 30 and 40 seconds. and plasma samples from patients on anticoagulant therapy may take up to perhaps 200 seconds to clot. It is obvious that recalcifying single test samples is an extremely tedious task. It has been found convenient in routine use to recalcify six or even eight tubes in quick succession if sufficient stop-watches are available. The organization required for this multiple testing is now described.

Enough brain and bovine plasma is pipetted out for about 24 tests. Diluted plasma samples from three patients are pipetted out in duplicate into six. of the tubes containing brain extract and adsorbed bovine plasma. The tubes containing the mixtures are then placed in the water-bath for three minutes before recalcification. Directly before the set time has elapsed the next set of test plasma samples are pipetted out. By this means it is possible to avoid any time wastage due to incubation: It must be emphasized that the test plasma should not be diluted more than 30 minutes before use, and that the bovine 
plasma and brain extract should also not be mixed more than 30 minutes before use.

B. Preparation of Dilution Curve.-The result is expressed as a "concentration" from a dilution curve plotted on double logarithmic paper. The preparation of this dilution curve is possibly the most important of the preparatory measures for this test. It has been found that different samples of normal plasma when diluted frequently give curves of different slopes even if the coagulation times of the undiluted plasmas are similar. Therefore all dilution curves used in this test are prepared from pooled normal plasma, the pool usually being a minimum of five normal samples. To maintain constant ionic strength all concentrations of the pooled plasma (except the $100 \%$ ) have to be diluted twice; it is essential that the dilutions are made as carefully as possible. The following concentrations are usually sufficient :

$100 \%$ (pooled plasma diluted $1 / 10$ in sol. II).

$50 \%$ (pooled plasma diluted $1 / 2$ in sol. I then $1 / 10$ in sol. II).

$20 \%$ (pooled plasma diluted $1 / 5$ in sol. I then $1 / 10$ in sol. II).

$10 \%$ (pooled plasma diluted $1 / 10$ in sol. I then $1 / 10$ in sol. II).

The above dilution curve should be made to test each new reagent (thromboplastin or bovine plasma). At least four estimations should be made on each concentration, and it is preferable to use two separate pooled samples of normal plasma. The mean coagulation times are plotted against " concentration" on double logarithmic graph paper, when a straight line should be obtained. The graph in routine use in this laboratory is shown in Fig. 2. It is particularly important to have multiple readings for the $10 \%$ and $20 \%$ dilutions of normal plasma because these show rather wide variations from one sample to another and the clotting times are much affected by small dilution errors.

It will be noted that on the graph the results are expressed simply as "concentration" as a percentage of normal. The method is not designed to measure any one substance, thus it is impossible to use specific terms. For routine reporting some phrase such as "one-stage prothrombin time by Owren's method, 105 seconds normal, 36 seconds $17 \%$ of normal" can be used.

C. Effect of Different Types of Adsorbed Bovine Plasma.- In the early stages of this investigation a comparison was made between three different methods of adsorbing the bovine plasma.

(a) Seitz filtration, when bovine plasma is passed through two grades of Seitz filter pad, containing $20 \%$ and $50 \%$ asbestos. The pads were manufactured by the Republic Seitz Filter Corp. of the U.S.A., the appropriate grades being G.P. for the first filtration and $\mathbf{S 3}$ for the second.

(b) Seitz filtration, as above except that Ford's "sterimats" were used. Grade F.C.B. was used for the first filtration and Grade SB for the second.

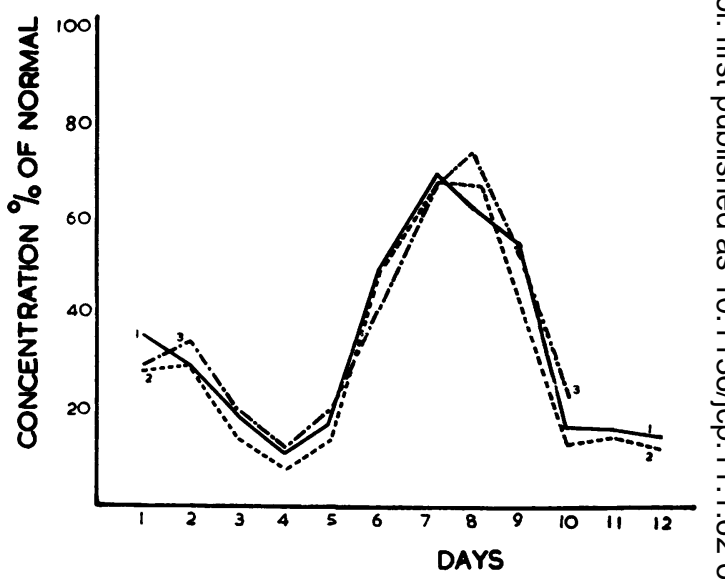

FIG. 1.-Daily estimations on a patient receiving " dindevan" using three different bovine plasma preparations: (1) Adsorption by Seitz filtration, (2) adsorption by $\mathrm{Ba}_{2} \mathrm{SO}_{4}$, (3) adsorption by Seitz filtration using Ford's " sterimats."

(c) Adsorption was achieved with barium sulphate (see above).

Patients being followed with Owren's method had daily estimations made with all three bovine plasma preparations. In all some 100 blood samples were tested, and no significant difference was found between the three preparations (Fig. 1). In this department the third method of adsorption was adopted as being the most suitable. In other depart- $\stackrel{\mathbb{Q}}{\mathscr{Q}}$ ments the filtration method may be found easier if $\underset{\vec{P}}{\vec{F}}$ sufficiently large filters are available.

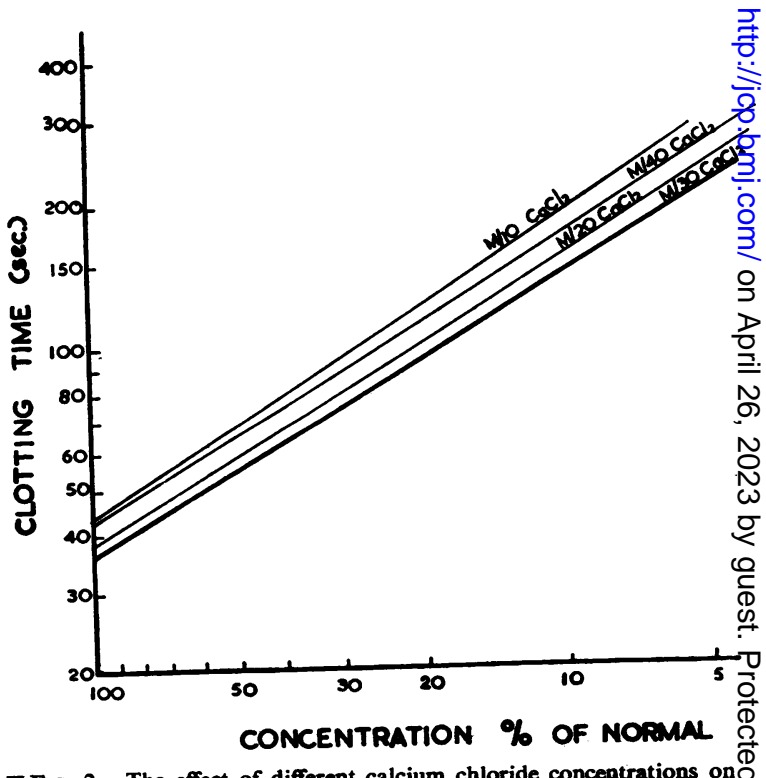

FIG. 2 The effect of different calcium chloride concentrations on the pooled normal plasma dilution curve. The thick line for $\mathrm{M} / 30 \mathrm{CaCl}_{2}$ is similar to the curve used routinely. 
FiG. 3.-Daily estimations on stored pooled normal plasma of the $100 \%$ and $50 \%$ levels. The dotted lines represent the $100 \%$ and $50 \%$ levels for the dilution curve in routine use at that time.

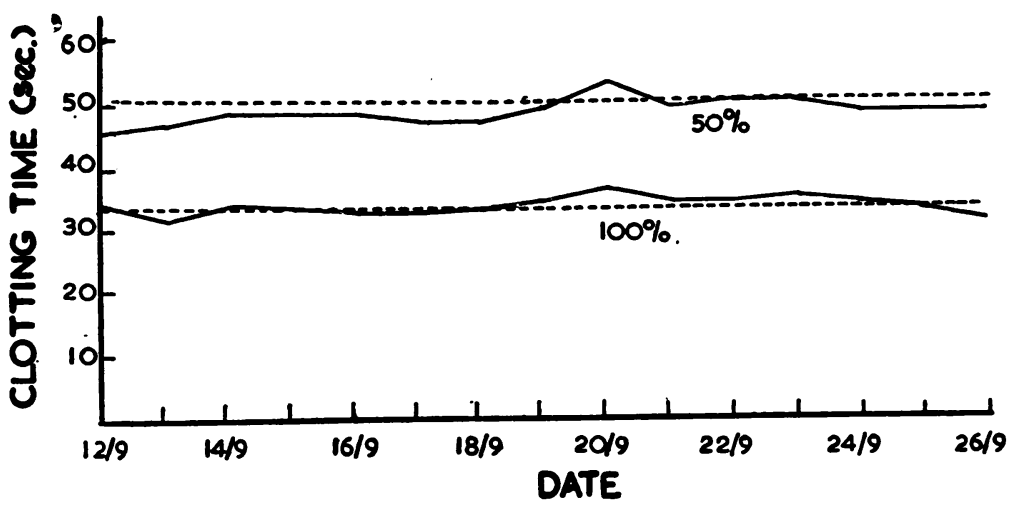

D. Effect of $\mathrm{CaCl}_{2}$ Concentration on Dilution Curve.-The optimum $\mathrm{CaCl}_{2}$ concentration is rather critical and may vary slightly for different batches of bovine plasma and brain extract. It is therefore of some importance that the optimum $\mathrm{CaCl}_{2}$ concentration is found for each new reagent. The concentrations used are $M / 20, M / 30$, and $M / 40$. Usually the $M / 30$ concentration gives the better times, though occasionally $M / 20$ has been selected. The effect of differing concentrations on the $100 \%$ and $50 \%$ levels of pooled normal plasma is usually quite small, whereas with the high dilutions of pooled normal plasma the effect will be more marked (Fig. 2).

E. Stability of Reagents over a Prolonged Period.-The bovine plasma and the brain extract appear to be stable over quite a prolonged period. No significant alteration has been detected over a period of three to four months. As it is unusual to prepare enough of these substances to last longer than this, tests over a longer period have not been made.

F. Daily Standardization of Method.-In routine use this method is very nearly self-standardizing from day to day. A steady supply of new patients assures the $100 \%$ level and usually there are a number of well stabilized patients, and by observing results on the samples from these patients any gross systematic deviation would be obvious. Also frequent estimations have been made of the $50 \%$ and $100 \%$ dilutions of stored pooled normal plasma. The results on the pooled samples have remained very constant (Fig. 3). Initially $10 \%$ and $20 \%$ dilutions were made daily, but this procedure gave rise to a good deal of unnecessary confusion because the clotting times of mixtures containing these high dilutions tended to be rather variable. The patient's plasma is always tested at the 1 in 10 dilution $(100 \%)$ and the higher dilutions of the dilution curve are determined on multiple samples when random variability is reduced to a minimum. It therefore seemed best to adhere to the procedure used by the originators of the technique and test daily only the 1 in 10 and 1 in 20 dilutions of the normal plasma (100\% and $50 \%)$.

G. Introduction of New Reagents. - It has been found that when a new batch of bovine plasma or thromboplastin or both is brought into routine use there is very little change in levels. Patients well stabilized should show only minimal alteration on the change-over. In routine practice daily estimations with the old and new reagents have been made for two or three days before the new reagent is adopted. The results given should always be in close agreement. An example of the sort of differences that may be expected in changing from one set of reagents to another is shown in Table $\mathrm{I}$.

TABLE I

EFFECT OF DIFFERENT REAGENTS

\begin{tabular}{|c|c|c|c|c|c|}
\hline & & & & \multicolumn{2}{|c|}{ Concentration $(\%)$ of Normal } \\
\hline \multicolumn{4}{|c|}{ Plasma } & $\begin{array}{l}\text { Thromboplastin I } \\
\text { Bovine Plasma I }\end{array}$ & $\begin{array}{l}\text { Thromboplastin II } \\
\text { Bovine Plasma II }\end{array}$ \\
\hline $\begin{array}{l}\text { James } \\
\text { Mores } \\
\text { Anderson } \\
\text { Morwood } \\
\text { Collins } \\
\text { Osborne } \\
\text { Howard } \\
\text { Finch }\end{array}$ & $\begin{array}{l}\cdots \\
\cdots \\
1 \\
\ldots \\
\cdots \\
\cdots\end{array}$ & $\begin{array}{l}\ldots \\
\ldots \\
\cdots \\
\cdots \\
\cdots \\
\cdots\end{array}$ & $\begin{array}{l}\ldots \\
\cdots \\
\cdots \\
\cdots \\
\cdots \\
\cdots\end{array}$ & $\begin{array}{l}23 \\
22 \\
29 \\
23 \\
30 \\
34 \\
88 \\
10\end{array}$ & $\begin{array}{l}21 \\
18 \cdot 5 \\
27 \\
21 \\
27 \\
28 \\
30 \\
9 \cdot 2\end{array}$ \\
\hline
\end{tabular}

\section{The Clinical Application of the Method}

The test is carried out daily until a stable level is reached and thereafter estimations are made on alternate days while the patient remains in hospital. If long-term treatment is given to out-patients the test is done at weekly or fortnightly intervals.

To establish the correct stabilizing dose there is no doubt that graphical representation of the results is helpful. General trends in the levels are much more obvious if seen graphically. A comparison between the results of Owren's method and the unmodified one-stage test is shown in Figs. 4 and 5. The anticoagulant dosage for both of these patients was calculated from the results of the unmodified one-stage test and Owren's method was at the time used experimentally. It is clear from a first impression that the two methods give roughly corresponding results. It is also clear that 


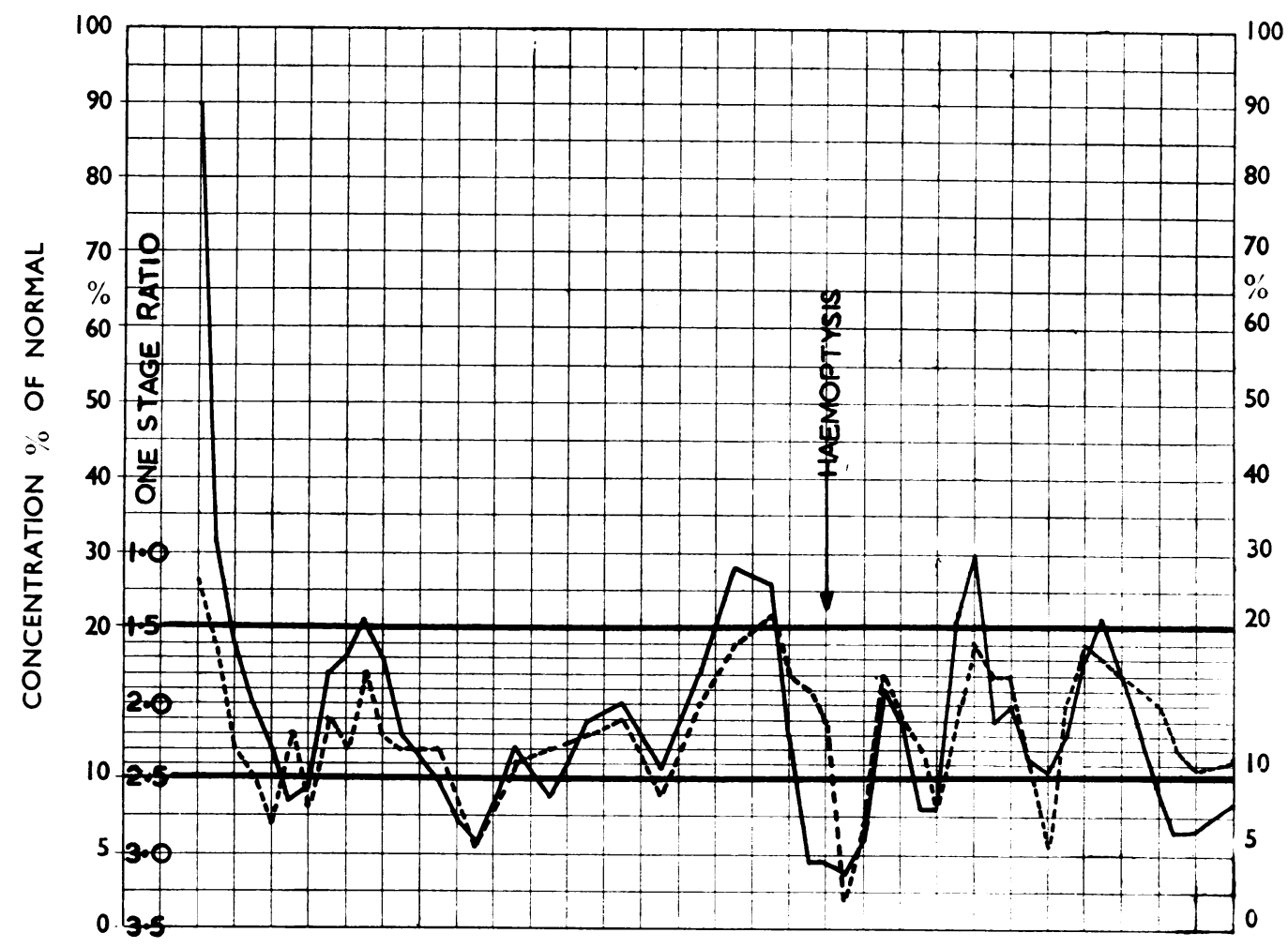

DOSAGE mgms. ANTICOAGULANT = DINDEVAN

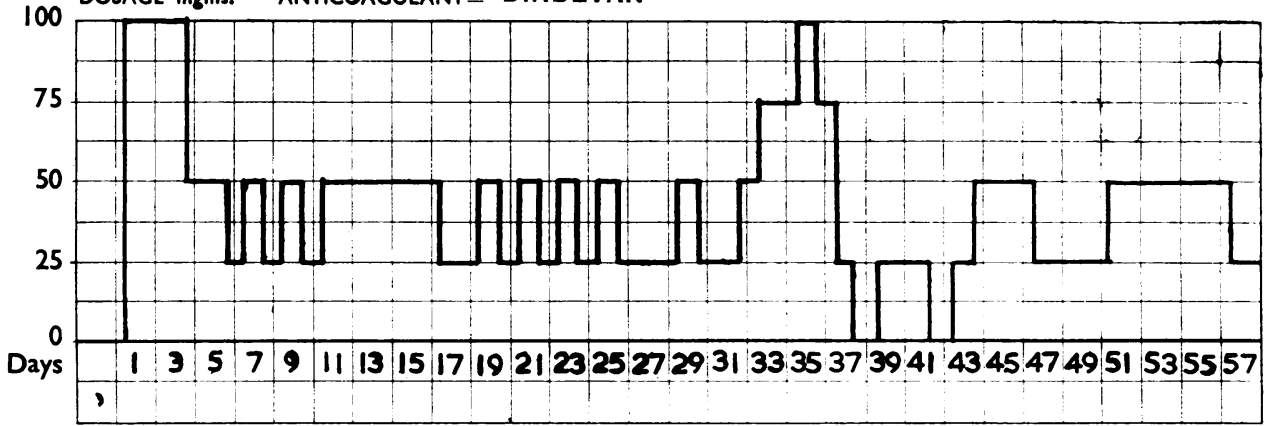

Fig. 4

Figs. 4 and 5. A comparison between Owren's method and the unmodified one-stage method on two patients receiving "dindevan." The unmodified one-stage method is expressed as a ratio $=\frac{\text { Patient's clotting time }}{\text { Normal clotting time }}$ The therapeutic range for both methods lies between the thick parallel lines. The charts shown are used in this hospital for graphing anticoagulant therapy data. Dotted line indicates one-stage ratio, the solid line Owren's method.

according to the results of Owren's method neither of the patients was ever established on an exactly balancing dose.

Detailed examination indicates the main sources of difficulty. In Fig. 4 it can be seen that from the eleventh to the sixteenth day of treatment the patient received $50 \mathrm{mg}$. of "dindevan" daily. The results of Owren's method show a slow but un $\stackrel{?}{+}$ mistakable downward trend. This finally becomes $\frac{0}{0}$ apparent on the unmodified method on the seven- $\overrightarrow{\mathbb{D}}$ teenth day when the dose was reduced. The dose $\frac{?}{\mathbb{Q}}$ was thereafter very nearly stabilized at about 37.5 을 $\mathrm{mg}$. daily. A single random low reading for the one-stage method led to reduction and then to a 


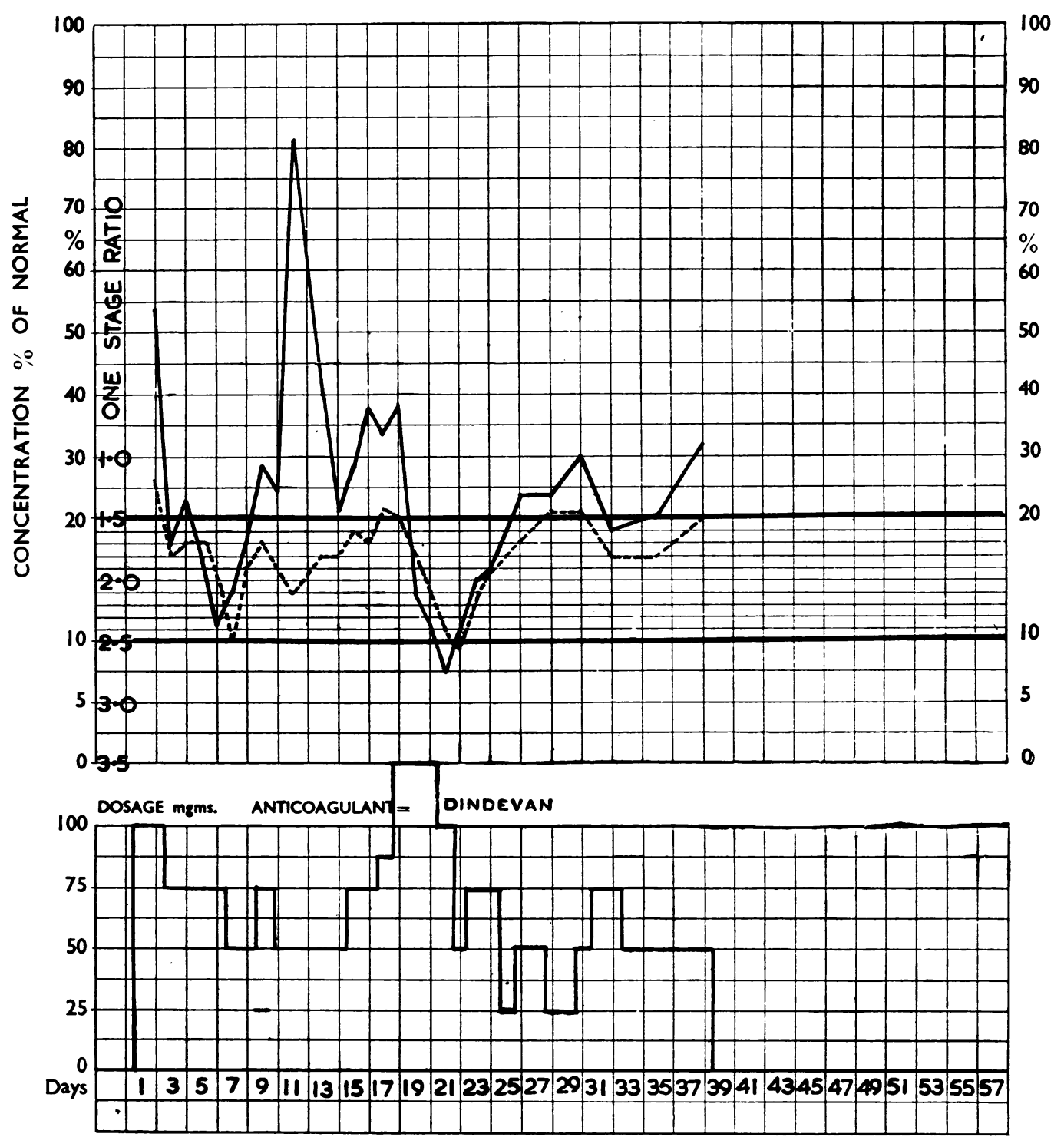

Fig. 5

very large and unjustified increase of dosage, the results of which are well reflected by the curve for Owren's method.

In Fig. 5 it is also clear that the failure to stabilize the dosage was mainly due to the very indefinite day-to-day trend of prothrombin times using the unmodified method. The balancing dose for this patient was probably of the order of 75 mg. daily.

The results obtained by any one-stage method reflect the dosage on the previous day with the addition of any drug cumulated from previous excessive doses. To reach a stabilizing dose it is therefore essential to apply an increasingly fine adjustment to the dose. Thus if $100 \mathrm{mg}$. doses are given on the first three days (Fig. 4) the effects of this treatment will be apparent in the results of testing on the fourth, fifth, and even sixth days. During this period an approximate dose is selected and, according to the general trend during the next four or five days, the dose may be altered by a small adjustment. Large dose alterations are very 
seldom necessary and always lead to serious imbalance in treatment (Figs. 4 and 5).

Detailed study of records such as these emphasizes the need for small dose adjustments. This is now possible since the introduction of the $10 \mathrm{mg}$. tablets of "dindevan." Using combinations of the 10 and $50 \mathrm{mg}$. tablets the dose may be adjusted to within $5 \mathrm{mg}$.

In the initial investigation of the method, patients receiving anticoagulant drugs had daily estimations made by both Quick's and Owren's method. About 1,500 blood samples from 63 patients were included in the survey. Almost invariably Owren's method showed the different trends in levels and hence dosage much sooner than did Quick's method. Also the day-to-day random variation was much smaller with Owren's method, making the trends much more obvious. The large random variation of Quick's method tended to mask rising or falling levels.

Owren's method has now been used for all anticoagulant work in this hospital group for 20 months. During this time nearly 12,000 estimations have been made, and the method has proved eminently satisfactory. The small random variation makes the method very suitable for out- patients receiving long-term therapy. It is also a $\overrightarrow{5}$ advantage in that blood specimens can be sent bP post with little effect on the result, making it pose sible for general practitioners to collect the samples in districts at some distance from the hospital.

\section{Summary}

A description is riven of the $P$ and $P$ (prothrombin and proconvertin) method of Owren aब্ used routinely in this department for the contros: of anticoagulant therapy. The method has bee found to be more sensitive and subject to less. random variation than Quick's one-stage method and to be particularly suitable for the control of long-term anticoagulant therapy.

I am grateful to Dr. Rosemary Biggs for her advice and encouragement during all stages of the prepara: tion of this paper.

$$
\text { REFERENCES }
$$

Astrup, T., and Müllertz, S. (1954). Schweiz. med. Wschr., 84, 81 and Hansen, J. R. (1951).Scand. J. clin. Lab. Invest ., 3, 20\$0

Biges, R., and Macfarlane, R. G. (1957). Human Blood Coagulation and Its Disorders, 2nd ed. Blackwell, Oxford.

Müllertz, S., and Storm, O. (1954). Circulation, 10, 213.

Owren, P. A. (1954). Schweiz. med. Wschr., 84, 822.

- and Aas, K. (1951). Scand. J. clin. Lab. Invest., 3, 201. 